\title{
Stress Analysis of Hole Orientation and Laminate Geometry Impacting on Boron/Epoxy Composites Laminates.
}

\author{
K.M. Alemu ${ }^{1}$, O.S. Fatoba ${ }^{2} ;$ D.K. Nageswara Rao ${ }^{3} ; E . T$. Akinlabi $^{4}$ \\ ${ }^{1}$ Faculty of Technology, Department of Mechanical Engineering, Debre Tabor University, Debre Tabor, Ethiopia. \\ ${ }^{2}$ Department of Mechanical Engineering Science, University of Johannesburg, Johannesburg, South Africa. \\ ${ }^{3}$ Faculty of Mechanical and Industrial Engineering, Bahir Dar Institute of Technology, Bahir Dar University, Bahir Dar, Ethiopia. \\ ${ }^{4}$ Pan African University for Life and Earth Sciences (PAULESI), Ibadan, Nigeria
}

\begin{abstract}
Boron/epoxy laminates are used in aircraft and space vehicles for their high strength. Evaluation of stresses and residual strength of the laminate with square cutout are not analyzed in the literature. The present work is focused on studying the effect of hole orientation and laminate geometry on Boron/Epoxy composites laminates under in-plane loading. The analytical solution for stresses around holes in laminates is derived using Savins's complex variables method to consider a multilayered plate with different hole shapes and orientations of loading. The basic equations of failure criteria available for plain laminates are derived to calculate the residual strength of the laminates with hole using the stresses obtained from the analytical solution. The derived analytical solution is validated by reproducing exactly the same results of earlier researchers even by other formulations and also by the results of finite element analysis using ANSYS. The $[0 / 0] \mathrm{s}$ laminate is not preferred due to highest stress concentrations at the corners that range between 12 to 12.45 . Similarly, [45/-45]s laminate is also not preferred due to its higher values of stress concentrations which range from 9.5 to 28 . The normalized stress for [0/90]s under $x$-axis loading is 9.6 and for $y$-axis loading it is 9.5 which is almost the same. Even for equi-biaxial loading, it is 8.5 and for shear loading, it is 12.45 . Except for shear loading, [0/90]s laminate seems to be a better choice for a reasonable value of stress concentration for any general case loading. The analytical solution derived in the present work is the most general and unique as it can yield the stresses around any shape of hole and laminate geometry and all types of in plane loading. This solution will be able to reproduce the results of all other solutions available in the literature by different formulations.
\end{abstract}

\subsection{Introduction}

Composite materials are very important in manufacturing processes because they are used widely in several areas such as aerospace industries and airframes and thanks to its remarkable strength and stiffness to weight ratios as well as its good resistance to corrosion and the fatigue $[1,2]$. Fiber reinforced polymeric (FRP) composites are nowadays widely used inengineering applications due to their outstanding features, such as high specific strength,specific stiffness, less/weight ratio and high structural performances, which are often theoutput of

* Corresponding author: proffatobasameni@gmail.com specific design and manufacturing strategies that aim to optimize the response of these composite structures to specific working conditions. Such superior mechanical properties of composite materials such as high stiffness and strength to weight ratios and

its attractive replacement for metallic materials made it possible for it to be used increasingly in many areas of technology including marine, aerospace, automotive and others. This has generated an increasing interest in the study of new possible design solutions aimed to enhance the performances of 
composite laminate structures under prescribed rules through the appropriate choice of materials, orientation, number of stacking sequence of layers that make up composite material and the determination of the optimal fiber orientation for each FRC layer [3-8].

Boron fibers, having about six times the modulus of elasticity of glass fibers and about the same strength and density as glass fibers, have been developed for use as reinforcements in structural plastics for aerospace vehicles and other high-performance applications. They are very stiff and have a high tensile and compressive strength. Boron fibers are used to repair cracked aluminum aircraft skins, because the thermal expansion of boron is close to aluminum and there is no galvanic corrosion potential [9-11]. Stress concentrations around cutouts are important from design point of view because they are generally the prime cause of failure under static loads. For most materials, the failure strength is strongly notch (or hole) sensitive [12, 13]. It has been experimentally validated that the net failure stress (considering the reduction in cross-sectional area due to cutouts) is generally much less than the ultimate tensile strength without any discontinuity [14].

Different cutout shapes in structural elements are needed to reduce the weight of thestructure or provide access to other parts of the structure. In some cases, structural elements are being damaged during their service life. It is well known that the presenceof a cutout or hole in a stressed member creates highly localized stresses at the vicinityof the cutout [15]. Uncoupling between extension and bending in symmetric laminatesmakes analyzing such laminates simpler. It also prevents a laminate fromtwisting due to thermal loads, such as cooling down from processing temperatures and temperature fluctuations during use such as in a space shuttle [1]. A major advantage of fibrous polymeric composites is that their anisotropy can be controlled through suitable choice of the influencing parameters. The unidirectional fiber reinforced composites provide much higher longitudinal mechanical properties compared to the transverse ones. Therefore, composite laminates are formed by stacking two or more laminas, with different fiber orientations, as to respond to complex states of stresses [1].

A major disadvantage of unidirectional composite materials is that they are highly anisotropic. One way around this is to form laminates by stacking two or more laminae' on top of each other with various orientations of the fiber direction and the mechanical properties are dependent upon the way the individual layers are stacked i.e. the stacking sequence. Predictions for cross-ply and angle-ply laminate mechanical properties are based upon classical lamination theory. Experimental data which indicates that the strength of symmetric composite laminates containing

identical ply orientations can be strongly dependent on the detailed stacking sequence, i.e., the actual arrangement of the various layers [16, 17]. One of the most important advantages of laminated composites is their potential to orient the laminas in different suitable directions or to choose the convenient stacking sequence configurations, such that the structural response to the complex state of stresses can be improved. It is evident that to enhance the mechanical properties of composite, controlling of the stacking sequence is very important $[18,19]$. The High stiffness and strength to weight ratios and its attractive replacement for metallic materials caused it to be used increasingly in many areas of technology including marine, aerospace, automotive and others. These Superior mechanical properties and extensive application of composite materials are achieved by tailoring of material properties through selective choice of orientation, number of stacking sequence of layers that make up composite material [20-22].

The present work addresses the determination of the stresses around square holes in symmetric boronepoxy unidirectional, cross ply and angle ply laminates under uni-axial and biaxial tensions and shear loading at infinity and to study the effect of orientation of square hole in $[0 / 0] \mathrm{s},[0 / 90] \mathrm{s}$, and $[45 /-$ $45] \mathrm{s}$ boron/epoxy symmetric laminates and type of in plane loading on the residual strength of the laminate.

\subsection{Methodology}

\subsection{Theoretical Formulations}

In the present work, a general solution is derived within the classical laminated plate theory to obtain two-dimensional stresses around holes in thin fiber reinforced composite laminates. The given hole problem is regarded as superposition of two subproblems corresponding to a sum of the respective complex potentials. Thus, by combining the two subproblems together will ensure the boundary conditions of no traction on the boundary of the hole while the plate is only under remote loading. Important feature of the present general solution is the introduction of generalized form of mapping function and also the arbitrary biaxial loading condition into the boundary conditions of the basic solution given by Savin [23] for in plane loading on thin anisotropic plates containing holes. 


\subsection{Generalized Mapping Function}

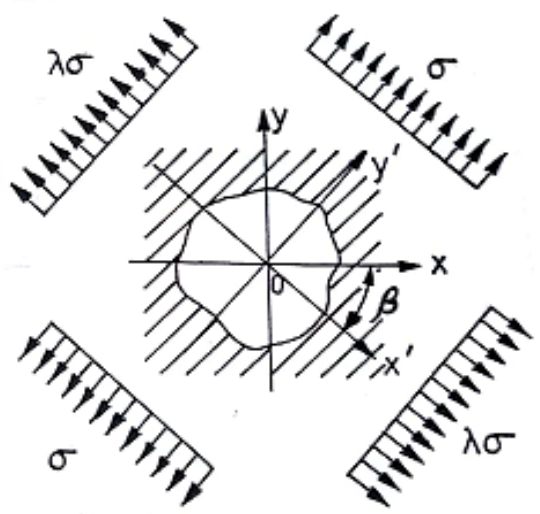

In the formulation of complex variable method using conformal mapping, it required to represent the area external to a given hole in the physical $z$-plane by the area outside the unit circle in $\zeta$-plane using a transformation function called the mapping function. Such a mapping function is given in a generalized form as seen in equation 1 :

$$
z=\omega(\zeta)=R\left(\zeta+\sum_{k=1}^{N} \frac{m_{k}}{\zeta^{k}}\right)
$$

where, $m_{k}$ are the constants of the mapping function. The solution is implemented by computer through coding in MATLAB. The solution is developed to consider a maximum number of terms $N$ equal to 1 to 19. To get the convergent results, higher number of terms in the mapping function are used. $R$ is a constant for the size of hole and since the stress distribution depends only on the shape but not on the size of the hole, the value of $R$ is taken as unity. For anisotropy, the equation shall be modified by introducing the complex parameters of anisotropy $s_{j}$. By affine transformation, the mapping function $z_{j}$ in equation 2 becomes:

$$
z_{j}=\omega_{j}(\zeta)=R\left[\left(\cos \theta+\sum_{k=1}^{N} m_{k} \cos k \theta\right)+s_{j}\left(\sin \theta-\sum_{k=1}^{N} m_{k} \sin k \theta\right)\right]
$$

By using the identities for sine and cosine and introducing them into Eq. (2), the mapping function in equation (2), is finally obtained in equation 3 as:

$$
z_{j}=\omega_{j}(\zeta)=\frac{R}{2}\left[a_{j}\left(\frac{1}{\zeta}+\sum_{k=1}^{N} m_{k} \zeta^{k}\right)+b_{j}\left(\zeta+\sum_{k=1}^{N} \frac{m_{k}}{\zeta^{k}}\right)\right]
$$

\subsection{Arbitrary Biaxial Loading Condition}

Arbitrary biaxial loading condition [24] is useful to consider various cases of in plane loading in to the boundary conditions. This is easily achieved by introducing the orientation angle $(\beta)$ and the biaxial loading factor $(\lambda)$ into the boundary conditions at infinity as seen in Figure 1.

Figure 1.Infinite Plate Containing Hole under Arbitrary Biaxial loading condition.

\subsection{Boundary Conditions at Infinity}

The boundary conditions about the arbitrary coordinate axes $x^{\prime} 0 y^{\prime}$ for each case of loading are given below for symmetric laminates within plane loading as seen in equation 4.

$$
\sigma_{x^{\prime}}^{\infty}=\lambda \sigma ; \quad \sigma_{y^{\prime}}^{\infty}=\sigma ; \quad \tau_{x^{\prime} y^{\prime}}^{\infty}=0 \quad \text { at }|z| \rightarrow \infty
$$

where, $\sigma^{\infty}{ }_{x \prime}$ and $\sigma^{\infty}{ }_{y}$ are applied stresses at infinity about the axis of $x^{\prime}$ and $y^{\prime}$ axes. Using the transformation relation of axes in equation 5 , it is given by:

$$
\begin{gathered}
\sigma_{x^{\prime}}^{\infty}+\sigma_{y^{\prime}}^{\infty}=\sigma_{x}^{\infty}+\sigma_{y}^{\infty} \\
\sigma_{y^{\prime}}^{\infty}-\sigma_{x^{\prime}}^{\infty}+2 i \tau_{x^{\prime} y^{\prime}}^{\infty}=\left(\sigma_{y}^{\infty}-\sigma_{x}^{\infty}+2 i \tau_{x y}^{\infty}\right) e^{2 i \beta}
\end{gathered}
$$

From Equations 4 and 5, the boundary conditions $\sigma^{\infty}{ }_{x}, \sigma^{\infty}{ }_{y}$ and $\tau^{\infty}{ }_{x y}$ about $x 0 y$ axes are explicitly written in equation 6 as:

$$
\begin{aligned}
\sigma_{x}^{\infty} & =\frac{\sigma}{2}[(\lambda+1)+(\lambda-1) \cos 2 \beta] \\
\sigma_{y}^{\infty} & =\frac{\sigma}{2}[(\lambda+1)-(\lambda-1) \cos 2 \beta](6) \\
\tau_{x y}^{\infty} & =\frac{\sigma}{2}[(\lambda-1) \sin 2 \beta]
\end{aligned}
$$

The boundary conditions in Equation 6 are useful to determine the stress functions of the hole free plate 


\subsection{Applications of Arbitrary Biaxial Loading Condition}

With refence figure 1 , the following values of $\lambda$ and $\beta$ shall be taken into Eqs. (6) to obtain different conditions of loading.

Inclined uni-axial tension: $\lambda=0, \beta \neq 0$

Loading along $x$-axis: $\lambda=0, \beta=\pi / 2$

Loading along $y$-axis: $\lambda=0, \beta=0$ Hydrostatic

tension: $\lambda=1, \beta \neq 0$

Equi-biaxial tension: $\lambda=1, \beta=0$

Shear stress $\lambda=-1, \beta=\pi / 4$ or $3 \pi / 4$

\subsection{General Solution for In-plane Loading of Symmetric Laminates}

\subsubsection{Complex Variable Formulation}

A thin anisotropic plate is considered under generalized plane stress condition. The thickness of plate $h$ is taken in $z$-direction and $x 0 y$ plane is taken as the mid plane of the plate. The plate is considered thin due to the value of $h / R$ being small, where, $R$ denotes the size of the hole. The stresses $\sigma_{z}, \tau_{y z}, \tau_{x z}$ are zero everywhere inside the plate in addition to being zero on top and bottom surfaces of the plate. From the generalized Hooke's law, the mean values of strains along the thickness of the plate, Airy's stress function $U(x, y)$, Compatibility equation and bi-harmonic equation, the characteristic equation can be given in equation 7 [25]:

$a_{11} s^{4}-2 a_{16} s^{3}+\left(2 a_{12}+a_{66}\right) s^{2}-2 a_{26} s+a_{22}=0$

And the roots in equation 8 are:

$$
\begin{array}{ll}
s_{1}=\alpha_{1}+i \beta_{1} & s_{2}=\alpha_{2}+i \beta_{2} \\
s_{3}=\alpha_{1}-i \beta_{1} & s_{4}=\alpha_{2}-i \beta_{2}
\end{array}
$$

$a_{i j}$ are compliance coefficients of the anisotropic plate., $(i, j=1,2,6)$

The stress components in terms of $\phi\left(z_{1}\right), \psi\left(z_{2}\right)$ and constants of anisotropy (s1 and s2) as [23, 26] are given in equation 9:

$$
\begin{aligned}
& \sigma_{x}=2 \operatorname{Re}\left[s_{1}^{2} \varphi^{\prime}\left(z_{1}\right)+s_{2}^{2} \psi^{\prime}\left(z_{2}\right)\right] \\
& \sigma_{y}=2 \operatorname{Re}\left[\varphi^{\prime}\left(z_{1}\right)+\psi^{\prime}\left(z_{2}\right)\right] \\
& \tau_{x y}=-2 \operatorname{Re}\left[s_{1} \varphi^{\prime}\left(z_{1}\right)+s_{2} \psi^{\prime}\left(z_{2}\right)\right]
\end{aligned}
$$

$\phi^{\prime}\left(z_{1}\right), \psi^{\prime}\left(z_{2}\right)$ are the first derivatives of the Mushkhelishvili's complex stress functions.

\subsubsection{Stress Functions for hole problems under Remote Loading}

\section{(a) Scheme of Solution}

The anisotropic plate with hole is applied by a remote tension $\sigma_{x^{\prime}}^{\infty}=\lambda \sigma, \sigma_{y^{\prime}}^{\infty}=\sigma$ at the outer edges as shown in Figure 2(c). The edges of the hole are free from loading. To determine the stresses around the hole, the solution is considered in two stages.

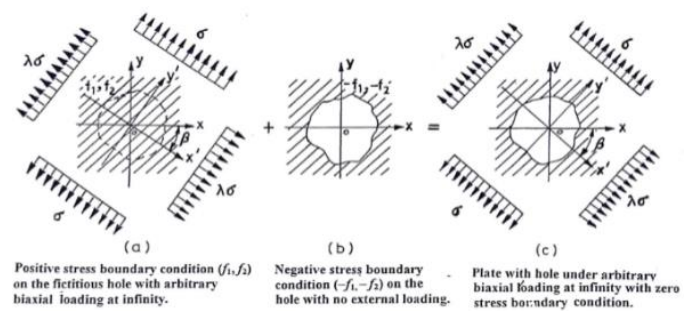

Figure 2. Scheme of solution for in plane loading of symmetric laminate with hole [27]

\section{(a1) First Stage Solution}

The stress functions $\phi_{l}\left(\mathrm{z}_{1}\right), \psi_{l}\left(\mathrm{z}_{2}\right)$, are obtained for the hole free plate due to applied stresses $\sigma_{x^{\prime}}^{\infty}, \sigma_{y^{\prime}}^{\infty}$ shown in Figure 2(a). From the stress functions $\phi_{1}\left(z_{1}\right)$, $\psi_{1}\left(z_{2}\right)$, the boundary conditions over the factious hole $\left(f_{1}, f_{2}\right)$ are determined.

\section{(a2) Second Stage Solution}

For the second stage solution, the plate with hole is applied by a negative of the boundary conditions $f_{1}, f_{2}$ on the hole boundary in the absence of remote loading as shown in Figure 2(b). The stress functions of the second stage solution $\phi_{o}\left(\mathrm{z}_{1}\right), \psi_{o}\left(\mathrm{z}_{2}\right)$ are determined from these boundary conditions. According to Savin's [23] method of superposition, stress functions $\phi\left(z_{1}\right)$, $\psi\left(z_{2}\right)$ for the given plate problem are obtained by adding the stress functions of the first and second stage solutions as shown in Figure 2(c). They are given in equation 10 as:

$$
\begin{aligned}
& \phi\left(z_{1}\right)=\phi_{1}\left(z_{1}\right)+\phi_{o}\left(z_{1}\right) \\
& \psi\left(z_{2}\right)=\psi_{1}\left(z_{2}\right)+\psi_{o}\left(z_{2}\right)
\end{aligned}
$$


By introducing the above stress functions $\phi\left(z_{1}\right), \psi\left(z_{2}\right)$ into Eqs.(11), the stresses $\sigma_{x}, \sigma_{y}, \tau_{x y}$ around the hole are obtained.

\section{(b) Stress Functions of First Stage Solution}

By taking $\phi_{1}^{\prime}\left(z_{1}\right)=\left(B^{*}+i C^{*}\right), \psi^{\prime}\left(z_{2}\right)=\left(B^{\prime *}+i C^{\prime *}\right)$ and upon integration, we get $\phi_{1}\left(z_{1}\right), \psi_{1}\left(z_{2}\right)$. The constant $C^{*}$ is associated with the rotation of the infinitely distant part of the plane $x 0 y$. Since no rotation is allowed and it results in $C^{*}$ to be zero as seen in equation 11.

$$
\begin{aligned}
& \phi_{1}\left(z_{1}\right)=B^{*} z_{1} \\
& \psi_{1}\left(z_{2}\right)=\left(B^{\prime *}+i C^{\prime *}\right) z_{2}
\end{aligned}
$$

\section{(c) Boundary Conditions from First Stage Solution}

The boundary conditions on the fictitious hole are given in equation 12 as:

$$
\begin{aligned}
& f_{1}=2 \operatorname{Re}\left[\phi_{1}\left(z_{1}\right)+\psi_{1}\left(z_{2}\right)\right] \\
& f_{2}=2 \operatorname{Re}\left[s_{1} \phi_{1}\left(z_{1}\right)+s_{2} \psi_{1}\left(z_{2}\right)\right]
\end{aligned}
$$

Now the plate with hole and negative of the boundary conditions obtained in the first stage solution such as: $f_{1}{ }^{0}=-f_{1}, \quad f_{2}{ }^{0}=-f_{2}$ are considered as shown in Figure 2(b). They are given in equation 13 as:

$$
\begin{aligned}
& f_{1}^{0}=-2 \operatorname{Re}\left[B^{*} z_{1}+\left(B^{\prime *}+i C^{* *}\right) z_{2}\right] \\
& f_{2}^{0}=-2 \operatorname{Re}\left[s_{1} B^{*} z_{1}+s_{2}\left(B^{\prime *}+i C^{\prime *}\right) z_{2}\right]
\end{aligned}
$$

The constants, $B^{*}, B^{\prime *}, C^{\prime *}$ in Equations 11 and 13 are given in equation 14 as:

$$
\begin{gathered}
B^{*}=\frac{\sigma_{x}^{\infty}+\left(\alpha_{2}^{2}+\beta_{2}^{2}\right) \sigma_{y}^{\infty}+2 \alpha_{2} \tau_{x y}^{\infty}}{2\left[\left(\alpha_{2}-\alpha_{1}\right)^{2}+\left(\beta_{2}^{2}-\beta_{1}^{2}\right)\right]} \\
B^{* *}=\frac{\left(\alpha_{1}^{2}-\beta_{1}^{2}-2 \alpha_{1} \alpha_{2}\right) \sigma_{y}^{\infty}-\sigma_{x}^{\infty}-2 \alpha_{2} \tau_{x y}^{\infty}}{2\left[\left(\alpha_{2}-\alpha_{1}\right)^{2}+\left(\beta_{2}^{2}-\beta_{1}^{2}\right)\right]} \\
C^{*}=\frac{\left\{\left(\alpha_{1}-\alpha_{2}\right) \sigma_{x}^{\infty}+\left[\alpha_{2}\left(\alpha_{1}^{2}-\beta_{1}^{2}\right)-\alpha_{1}\left(\alpha_{2}^{2}-\beta_{2}^{2}\right)\right] \sigma_{y}^{\infty}+\left[\left(\alpha_{1}^{2}-\beta_{1}^{2}\right)-\left(\alpha_{2}^{2}-\beta_{2}^{2}\right)\right] \tau_{x y}^{\infty}\right\}}{2 \beta_{2}\left[\left(\alpha_{2}-\alpha_{1}\right)^{2}+\left(\beta_{2}^{2}-\beta_{1}^{2}\right)\right]}
\end{gathered}
$$

Upon introducing the mapping function (3) into the boundary conditions (13), we have equations 15 and 16:

$$
\begin{aligned}
& f_{1}^{0}=-\left[\left(K_{1}+\bar{K}_{2}\right)\left(\frac{1}{\zeta}+\sum_{k=1}^{N} m_{k} \zeta^{k}\right)+\left(K_{2}+\bar{K}_{1}\right)\left(\zeta+\sum_{k=1}^{N} \frac{m_{k}}{\zeta^{k}}\right)\right] \\
& f_{2}^{0}=-\left[\left(K_{3}+\bar{K}_{4}\right)\left(\frac{1}{\zeta}+\sum_{k=1}^{N} m_{k} \zeta^{k}\right)+\left(K_{4}+\bar{K}_{3}\right)\left(\zeta+\sum_{k=1}^{N} \frac{m_{k}}{\zeta^{k}}\right)\right]
\end{aligned}
$$

where,

$$
\begin{aligned}
& K_{1}=\frac{R}{2}\left[B^{*} a_{1}+\left(B^{\prime *}+i C^{*}\right) a_{2}\right] \\
& K_{2}=\frac{R}{2}\left[B^{*} b_{1}+\left(B^{\prime *}+i C^{\prime *}\right) b_{2}\right] \\
& K_{3}=\frac{R}{2}\left[s_{1} B^{*} a_{1}+s_{2}\left(B^{\prime *}+i C^{\prime *}\right) a_{2}\right] \\
& K_{4}=\frac{R}{2}\left[s_{1} B^{*} b_{1}+s_{2}\left(B^{\prime *}+i C^{\prime *}\right) b_{2}\right]
\end{aligned}
$$

\section{(d) Stress Functions of Second Stage Solution}

Introducing the boundary conditions in equation 15 into the Schwarz formula Eq (4) of Savin [23], the stress functions for the second stage are obtained using the following in equation 17 :

$$
\begin{aligned}
& \phi_{0}(\zeta)=\frac{i}{4 \pi\left(s_{1}-s_{2}\right)} \int_{\gamma}\left(s_{2} f_{1}^{0}-f_{2}^{0}\right) \frac{t+\zeta}{t-\zeta} \frac{d t}{t}+\lambda_{1} \\
& \psi_{0}(\zeta)=\frac{-i}{4 \pi\left(s_{1}-s_{2}\right)} \int_{\gamma}\left(s_{1} f_{1}^{0}-f_{2}^{0}\right) \frac{t+\zeta}{t-\zeta} \frac{d t}{t}+\lambda_{2}
\end{aligned}
$$

where, $\gamma$ is the boundary of the unit circle in $\zeta$-plane $\lambda_{1}, \lambda_{2}$ are imaginary constants which will not affect the stresses and may be dropped further. The following results are used while evaluating the integrals in equation 18:

$\int_{\gamma} \frac{1}{t^{k}} \cdot \frac{t+\zeta}{t-\zeta} \cdot \frac{d t}{t}=\frac{4 \pi i}{\zeta^{k}} \quad \int_{\gamma} t^{k} \cdot \frac{t+\zeta}{t-\zeta} \cdot \frac{d t}{t}=0$

Finally, the stress functions of the second stage solution $\phi_{o}(\zeta), \psi_{o}(\zeta)$ from equation 17 are obtained using equations 19 and 20

$$
\left.\begin{array}{l}
\phi_{0}(\zeta)=\left[\begin{array}{ll}
\frac{a_{3}}{\zeta}+b_{3} & \sum_{k=1}^{N} \frac{m_{k}}{\zeta^{k}}
\end{array}\right] \\
\psi_{0}(\zeta)=-\left[\frac{a_{4}}{\zeta}+b_{4} \sum_{k=1}^{N} \frac{m_{k}}{\zeta^{k}}\right.
\end{array}\right]
$$

where, 


$$
\begin{aligned}
& a_{3}=\frac{1}{s_{1}-s_{2}}\left[s_{2}\left(K_{1}+\bar{K}_{2}\right)-\left(K_{3}+\bar{K}_{4}\right)\right] \\
& b_{3}=\frac{1}{s_{1}-s_{2}}\left[s_{2}\left(K_{2}+\bar{K}_{1}\right)-\left(K_{4}+\bar{K}_{3}\right)\right] \\
& a_{4}=\frac{1}{s_{1}-s_{2}}\left[s_{1}\left(K_{1}+\bar{K}_{2}\right)-\left(K_{3}+\bar{K}_{4}\right)\right] \\
& b_{4}=\frac{1}{s_{1}-s_{2}}\left[s_{1}\left(K_{2}+\bar{K}_{1}\right)-\left(K_{4}+\bar{K}_{3}\right)\right]
\end{aligned}
$$

\subsubsection{Final Solution for Stresses}

Stress functions $\phi_{1}\left(z_{1}\right), \psi_{1}\left(z_{2}\right)$ and $\phi_{o}\left(z_{1}\right), \psi_{o}\left(z_{2}\right)$ are inserted into equation 10 and by superposition, the stress functions $\phi\left(z_{1}\right), \psi\left(z_{2}\right)$ for the given plate problem are obtained in equations 21 and 22 .

$$
\begin{aligned}
& \sigma_{x}=\sigma_{x}^{\infty}+2 \operatorname{Re}\left[s_{1}^{2} \phi_{0}^{\prime}\left(z_{1}\right)+s_{2}^{2} \psi_{0}^{\prime}\left(z_{2}\right)\right] \\
& \sigma_{y}=\sigma_{y}^{\infty}+2 \operatorname{Re}\left[\phi_{0}^{\prime}\left(z_{1}\right)+\psi_{0}^{\prime}\left(z_{2}\right)\right] \\
& \tau_{x y}=\tau_{x y}^{\infty}-2 \operatorname{Re}\left[s_{1} \phi_{0}^{\prime}\left(z_{1}\right)+s_{2} \psi_{0}^{\prime}\left(z_{2}\right)\right]
\end{aligned}
$$

\begin{tabular}{|c|c|c|c|}
\hline $\begin{array}{l}\text { Laminate } \\
\text { geometry }\end{array}$ & $\begin{array}{c}\text { Type } \\
\text { of } \\
\text { loading }\end{array}$ & $\begin{array}{c}\text { Maximum } \\
\text { von-Mises } \\
\text { stress(normalized) }\end{array}$ & $\begin{array}{c}\text { locations of } \\
\text { maximum stress }\end{array}$ \\
\hline$[0 / 0] \mathrm{s}$ & \multirow{3}{*}{$\begin{array}{l}x \text {-axis } \\
\text { loading }\end{array}$} & 12 & $\begin{array}{l}50^{0}, 130^{0}, \\
230^{0}, 310^{0}\end{array}$ \\
\hline$[0 / 90] \mathrm{s}$ & & 9.5 & $50^{\circ}, 130^{\circ}, 230^{\circ}$ \\
\hline$[45 /-45] \mathrm{s}$ & & 14 & $45^{0}, 135^{0}, 315^{0}$ \\
\hline$[0 / 0] \mathrm{s}$ & \multirow{3}{*}{$\begin{array}{l}y \text {-axis } \\
\text { loading }\end{array}$} & 6.8 & $45^{0}, 135^{0}, 225^{0}, 315^{0}$ \\
\hline$[0 / 90] \mathrm{s}$ & & 9.6 & $40^{\circ}, 140^{\circ}, 320^{\circ}$ \\
\hline$[45 /-45] \mathrm{s}$ & & 14 & $45^{\circ}$ \\
\hline$[0 / 0] \mathrm{s}$ & \multirow{3}{*}{$\begin{array}{l}\text { equi- } \\
\text { biaxial } \\
\text { loading }\end{array}$} & 8.7 & $315^{\circ}$ \\
\hline$[0 / 90] \mathrm{s}$ & & 8.5 & $50^{\circ}, 140^{0}, 230^{\circ}$ \\
\hline$[45 /-45] \mathrm{s}$ & & 28 & $\begin{array}{c}45^{0}, 135^{0} \\
225^{0}, 315^{0}\end{array}$ \\
\hline$[0 / 0] \mathrm{s}$ & \multirow{3}{*}{$\begin{array}{l}\text { shear - } \\
\text { loading }\end{array}$} & 12.45 & $\begin{array}{l}45^{0}, 135^{0} \\
225^{0}, 315^{0}\end{array}$ \\
\hline$[0 / 90] \mathrm{s}$ & & 12.45 & $\begin{array}{c}45^{0}, 135^{0}, \\
225^{0}, 315^{0}\end{array}$ \\
\hline$[45 /-45] \mathrm{s}$ & & 11.6 & $\begin{array}{l}45^{0}, 135^{0}, \\
225^{0}, 315^{0}\end{array}$ \\
\hline
\end{tabular}

where,

$$
\begin{array}{ll}
\phi_{0}^{\prime}\left(z_{1}\right)=\frac{\phi_{0}^{\prime}(\zeta)}{\omega_{1}^{\prime}(\zeta)} & \psi_{0}^{\prime}\left(z_{2}\right)=\frac{\psi_{0}^{\prime}(\zeta)}{\omega_{2}^{\prime}(\zeta)} \\
\omega_{1}^{\prime}(\zeta)=\frac{d z_{1}}{d \zeta} & \omega_{2}^{\prime}(\zeta)=\frac{d z_{2}}{d \zeta}
\end{array}
$$

Table 1: Maximum values of von-Mises stress for boronepoxy laminates with square hole normal sides.
Applying the transform from Cartesian co-ordinates $x 0 y$ into orthogonal curvilinear co-ordinates $\rho=$ const., $\theta=$ const, the stresses given by equation 21 in Cartesian coordinates are transformed to orthogonal curvilinear coordinate system $(\rho, \theta)$ by means of the following relations in equation 23 :

$$
\begin{aligned}
& \sigma_{\theta}+\sigma_{\rho}=\sigma_{y}+\sigma_{x} \\
& \sigma_{\theta}-\sigma_{\rho}+2 i \tau_{\rho \theta}=\left(\sigma_{y}-\sigma_{x}+2 i \tau_{x y}\right) e^{2 i \alpha}
\end{aligned}
$$

\subsection{Results and discussion}

\subsection{Normalised Von Misses Stresses Around Square Hole with Normal Sides}

The effect of the orientation of square hole with normal sides in boron/epoxy laminates with different stacking sequences of $[0 / 0]_{\mathrm{s}},[0 / 90]_{\mathrm{s}}$, and $[45 /-45]$ is subjected to unidirectional loading in $x, y$-directions and equibiaxial loading and shear stress at infinity. The results for stresses around the boundary of square hole with normal sides in boron-epoxy laminates of $[0 / 0] \mathrm{s},[0 / 90] \mathrm{s}$ and $[45 /-45] \mathrm{s}$ geometry under remote uniaxial loading in $x, y$-directions and equi-biaxial loading and shear stress are presented in Figures 3 14. The results obtained by the present solution are validated by finite element method for each case. It is noted that the results by these two methods have close agreement indicating the reliability of the results of the present solution. A summary of the results is presented in Table 1 for all the cases considered in Figures 3 - 14.

It is noted from the results in Table 1, that a lowest stress concentration equal to 6.8 is present for $[0 / 0] \mathrm{s}$ laminate under $y$-axis loading, i.e., the loading is normal to the direction of fibers. On the contrary, a higher stress concentration equal to 12 is obtained for the same laminate when the loading is in the direction of fibers, i.e., along $x$-axis. The higher value obtained can be attributed to the prevailing discontinuity of fibers in $x$-direction along the vertical edges on either side of the hole. There is a shearing interface near the corners due to discontinuity of fibers in the direction of loading which results in mode-II fracture of the fiber-matrix interface which has only a weaker interaction of shear resistance to offer against the applied tension. Since the shear resistance of the interface is less, a high stress concentration is obtained near the corner.

This result is in conformation with the observation by Jong [28] that shear stress concentration is more 
relevant in anisotropic plates rather than the tangential or von-Mises stress. When the same [0/0]s laminate is applied by $y$-axis loading, the normalized stress is only 6.8 which is close to one-half of the previous value. This lower value indicates the presence of a tensile condition of mode-I fracture of fiber matrix interface near the corners due to loading in $y$-direction. The lower stress concentration can be attributed to the higher resistance offered by interfaces with a tensile resistance which is normally higher. Hence, there is a lower value of stress concentration for loading along $y$-axis.

Another feature of a shift of $5^{\circ}$ on either side of the corner can be noted for $x$ - and $y$-axes loadings. It is at $50^{\circ}$ for $x$-axis loading while it is at $40^{\circ}$ for $y$-axis loading. This shift is oriented away from the direction of loading which can be attributed to the shearing of interface between the broken and unbroken fibers near the corners with a stretch of the fibers along the edges in the direction of loading while there will be a sliding of the broken fibers towards the empty space of the hole due to shearing action. It concludes that orientation of fibers normal to the direction of loading has reduced the stress concentration with $5^{\circ}$ shift from the corners in the direction of fibers which applies for the $[0 / 0]$ s laminate under $y$-axis loading.

The normalized stress for [0/90]s under $x$-axis loading is 9.6 and for $y$-axis loading it is 9.5 which is almost the same. Even for equi-biaxial loading, it is 8.5 and for shear loading, it is 12.45. Except for shear loading, [0/90]s laminate seems to be a better choice for a reasonable value of stress concentration for any general case loading. A cursory look at the results of [45/-45]s laminates for different types of loading indicates that there is higher value of stress concentration for all cases of normal loads. The highest value is 28 for [45/-45]s laminate under equibiaxial loading, while it is 14 for both $x$-and $y$-axis loadings. This is due to the fibers being oriented at $45^{\circ}$ to the directions of loading and only matrix must be sharing the maximum load. This indicates a weaker resistance offered by the interfaces due to fibers oriented at an angle and not contributing much for load sharing. Hence, [45/-45]s geometry is a bad choice especially when the direction of loading is along $x$-, $y$ or equi-biaxial directions. However, for shear loading, the stress concentration is 11.6 which is lower compared to the values under loading in normal directions.

Generally, shear loading has caused a higher stress concentration at the corner and the material around the hole boundary is subjected to higher severity of loading compared to other types of loading. From the study of the values of normalized von-Mises stresses around the square hole with normal sides in a boronepoxy unidirectional $[0 / 0] \mathrm{s}$, cross-ply $[0 / 90] \mathrm{s}$ and angle ply [45/-45]s laminates under various cases of remote loading, it is inferred that the $[0 / 90] \mathrm{s}$, cross ply laminate is a better choice for all cases of loading.

\subsubsection{Results for Laminates with square hole- normal sides under $x$-axis loading}

\subsubsection{1 [0/0]s Laminate}

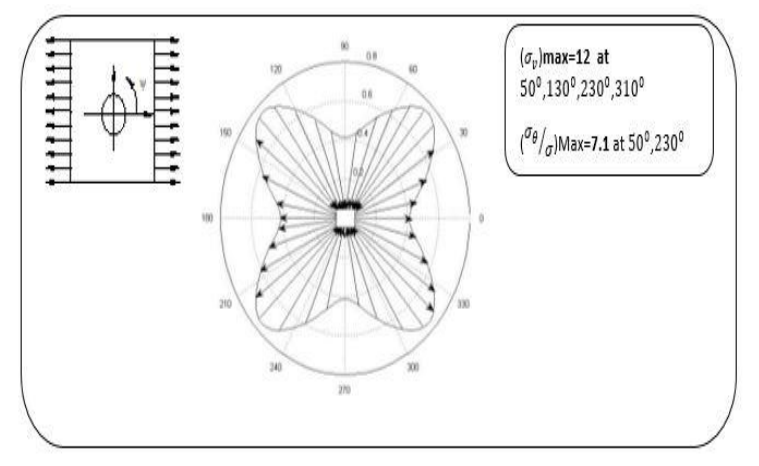

(a)

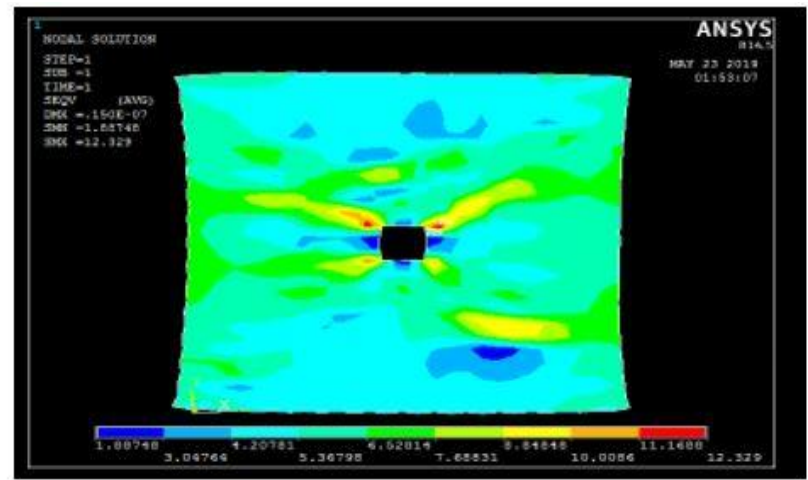

(b)

Figure 3: Stress values for [0/0]s Laminate with normal square hole under $\mathrm{x}$-axis loading. a) Present solution b) ANSYS result 


\subsubsection{2. [0/90]s Laminate}

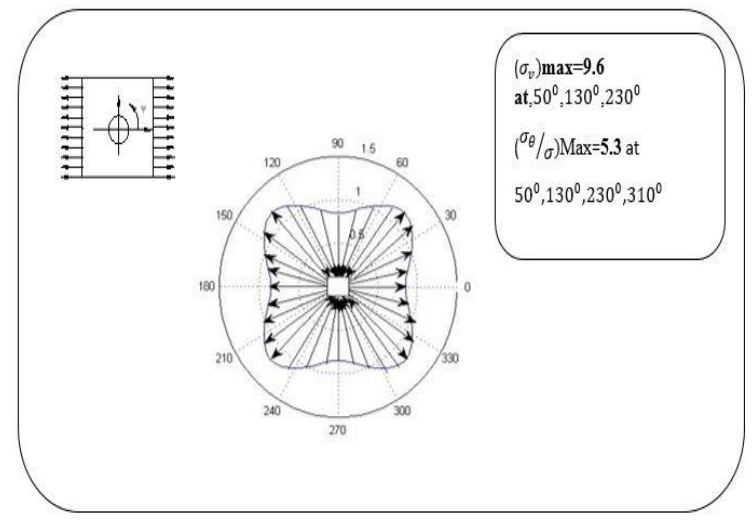

(a)



(b)

Figure 4: Stress values for [0/90]s Laminate with normal square hole under $\mathrm{x}$-axis loading. (a) Present solution b) ANSYS result

\subsubsection{3. [ \pm 45$]$ s Laminate}



(a)



(b)

Figure 5: Stress values for $[ \pm 45]$ s laminate with normal Square Hole under x- loading. (a) Present solution (b) ANSYS result

3.1.2 Results for Laminates with square hole-normal sides under y-axis loading

\subsubsection{1 [0/0]s Laminate}

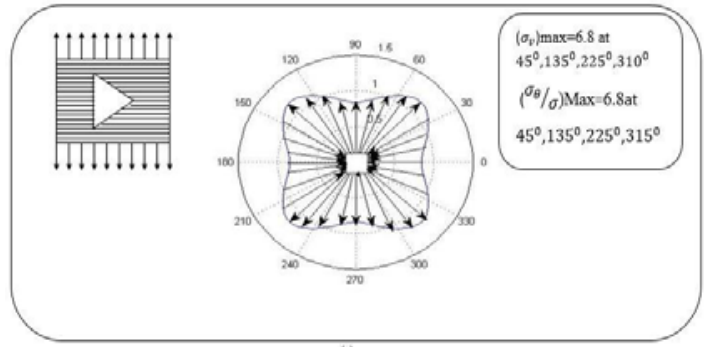

(a)

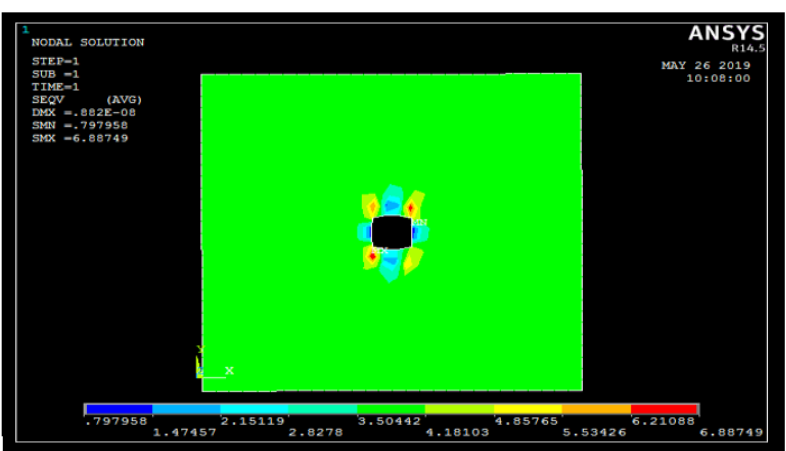

(b)

Figure 6: Stress values for $[0 / 0]$ s Laminate with normal square hole under y-axis loading. (a) Present solution (b) ANSYS result

\subsubsection{2 [0/90]s Laminate}




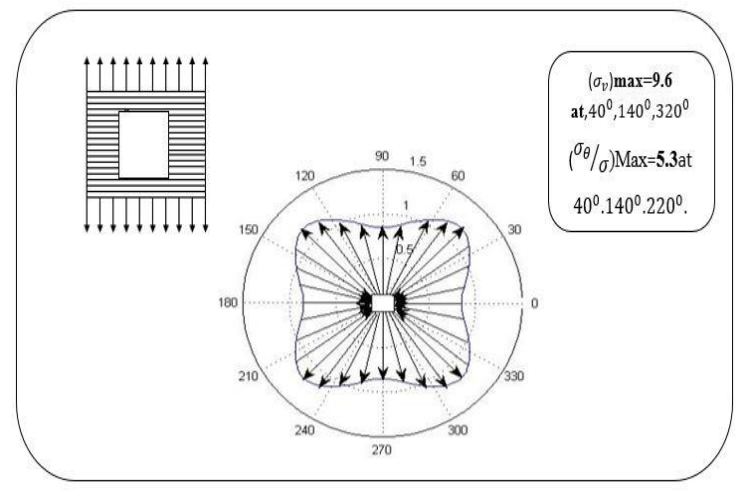

(a)



(b)

Figure 7: Stress Values for [0/90]s laminate with normal square hole under y-axis loading a) Present solution b) ANSYS result

\subsubsection{3 [45/-45] s Laminate}

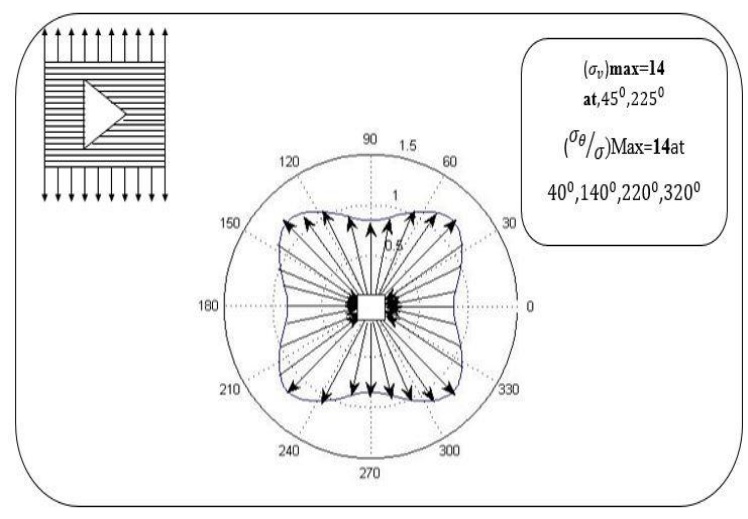

(a)

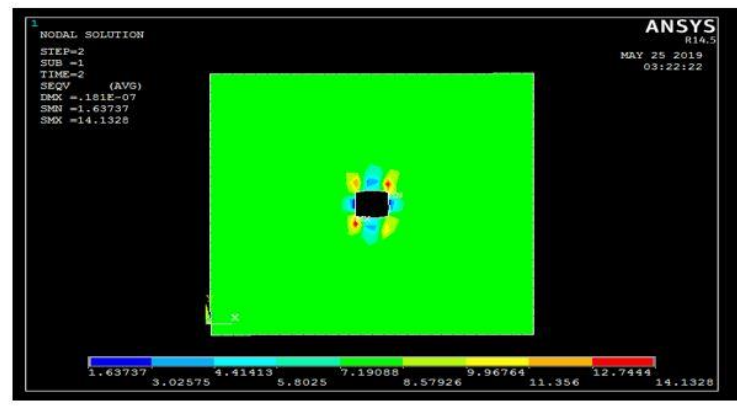

(b)

Figure 8: Stress Values for $\left[ \pm 45^{0}\right]$ s laminate with normal square hole under y- loading. a) Present solution b) ANSYS result

\subsubsection{Results for Laminates with Square Hole- Normal Sides under equi-biaxial Loading}

\subsubsection{1. [0/0]s Laminate}

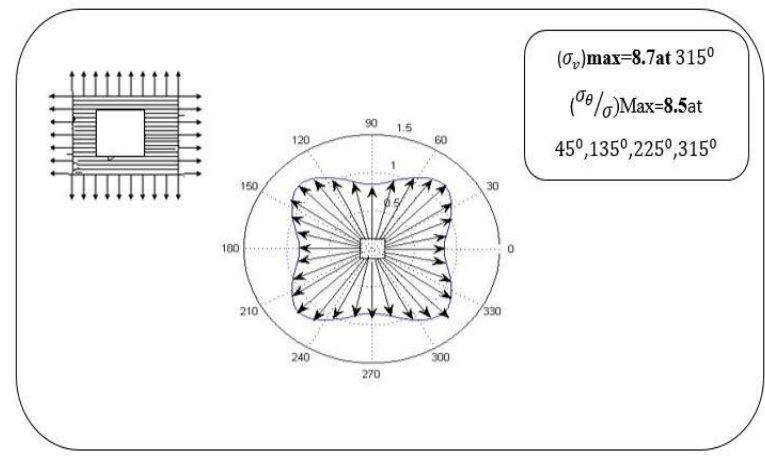

(a)



(b)

Figure 9: Stress Values for [0/0]s Laminate with normal Square Hole under equi-bi axial Loading. a) Present solution b) ANSYS result 


\subsubsection{2 [0/90]s Laminate}



(a)

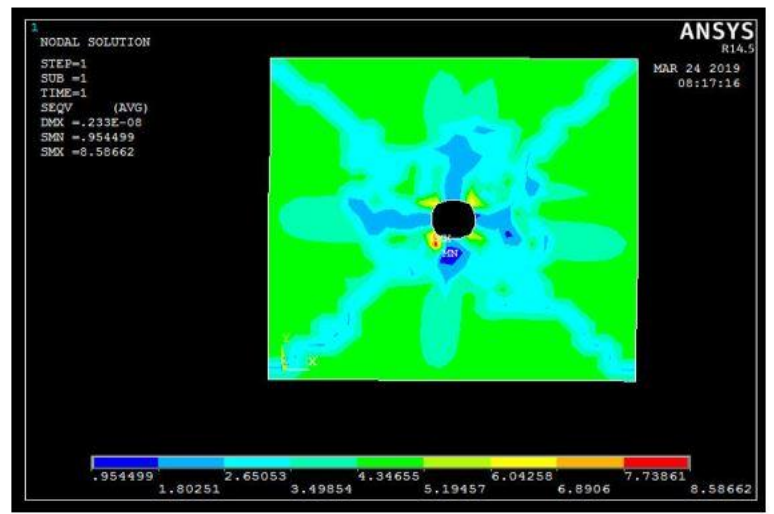

(b)

Figure 10: Stress Values for [0/90]s laminate with normal square hole under equi-biaxial loading a) Present solution b) ANSYS result

\subsubsection{3. [45/-45]s Laminate}

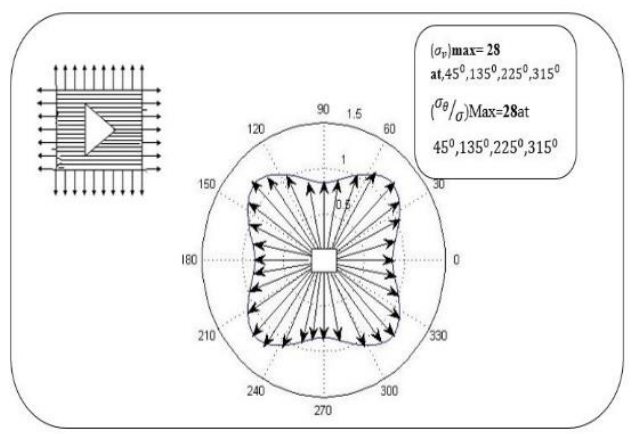

(a)

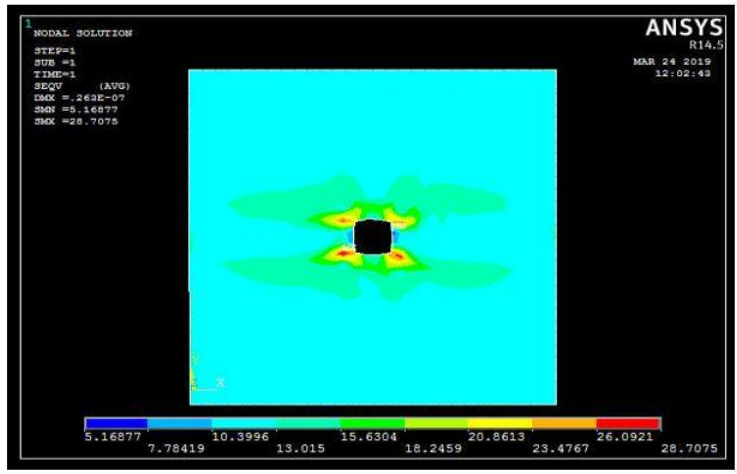

(b)

Figure 11: Stress Values for $\left[ \pm 45^{0}\right]$ s laminate with normal square hole under equi-biaxial loading. a) Present solution b) ANSYS result

\subsubsection{Results for Laminates with Square Hole- Normal Sides under Shear Loading}

\subsubsection{1. [0/0]s Laminate}

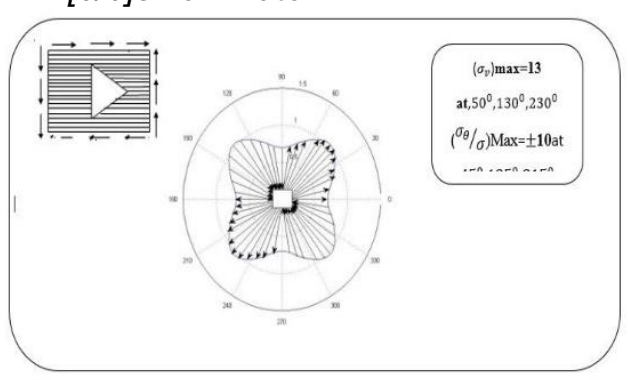

(a)

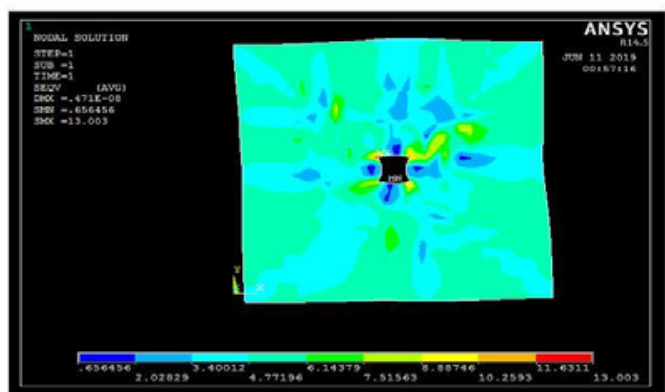

(b)

Figure 12: Stress Values for [0/0] s Laminate with Normal Square Hole under Shear Loading. a) Present solution b) ANSYS result 


\subsubsection{2. [0/90] s Laminate}

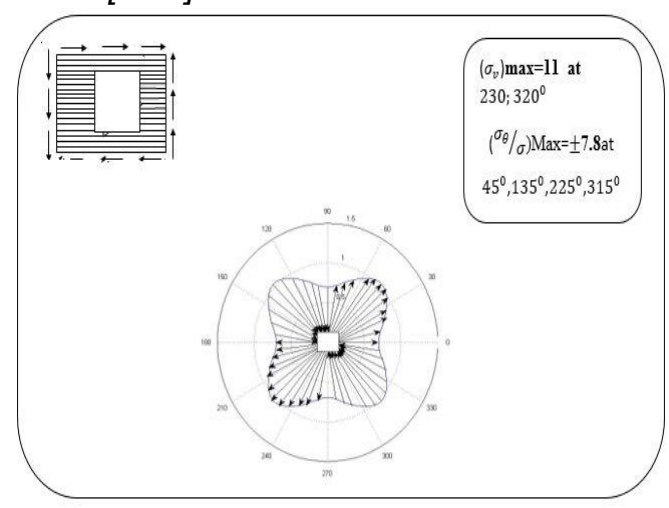

(a)

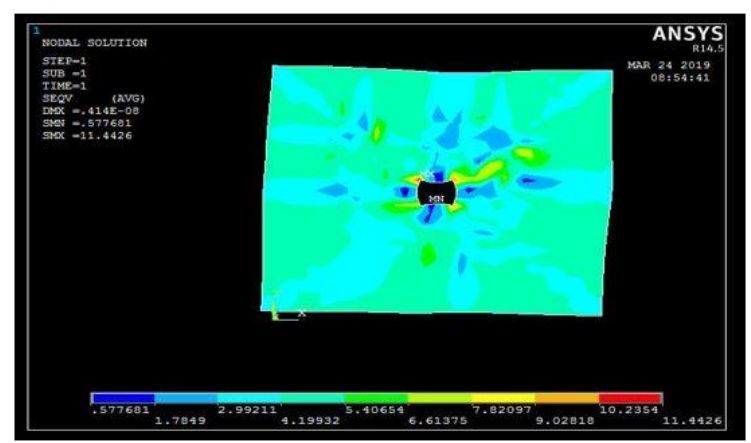

(b)

Figure 13: Stress Values for [0/90]s laminate with normal square hole under shear loading. a) Present solution b) ANSYS result

\subsubsection{3. [45/-45]s Laminate}

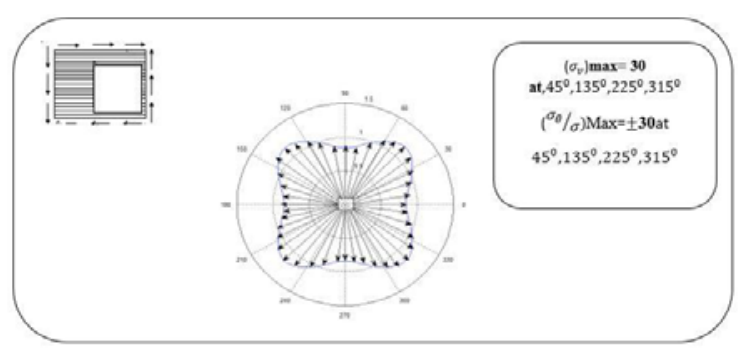

(a)

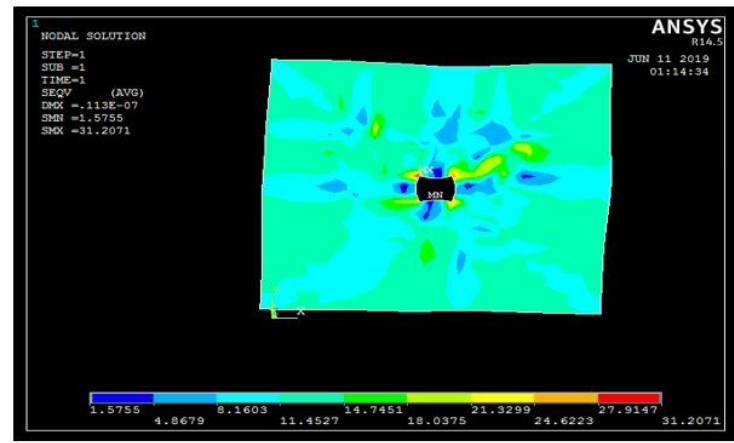

(b)

Figure 14: Stress Values for $\left[ \pm 45^{0}\right]$ s laminate with normal square hole under shear loading. a) Present solution b) ANSYS result

The square hole with normal sides in [0/90]s laminate gave moderate value of stress concentration for all the four types of in plane loading. The normalized vonMises stresses are equal to 9.5, 9.6, 8.5 and 12.45 under $x$-, $y$-, equi-biaxial and shear loading at infinity respectively. This indicates as a better choice of laminate for all types of in plane loading. Presence of fibers in the direction normal to the direction of loading in a laminate has resulted in reduction of stress concentration values that range between 6 and 12 for loading in $x-, y$-, and equi-biaxial directions on $[0 / 0] \mathrm{s}$, [0/90]s laminates while for [45/-45]s laminate, they are higher equal to 14,14 , and 28 for respective cases of loading. Moderate values of stress concentration equal $12.45,12.45$ and 11.6 are noted for $[0 / 0] \mathrm{s}$, [0/90]s and [45/-45]s laminates under shear loading. An insight into the values of normalized stresses at the corners for square hole with normal sides indicates a strong bearing on fiber matrix interaction at the corner zone. The normalized stresses are lower if there exists a tensile condition and they are higher if it is a purely a shear interface. Moderate values equal to 12.45 , 12.45 and 11.6 for shear loading on $[0 / 0] \mathrm{s},[0 / 90] \mathrm{s}$ and [45/-45]s respectively are present when a combination of both tensile and shear conditions exist at the interfaces.

- Presence of fibers in the direction normal to the direction of loading gave lowest value of stress as in the case $[0 / 0] \mathrm{s}$ laminate under $y$-axis loading has produced a normalized stress equal to 6.8 .

- A cross ply [0/90]s laminate gave moderate values of stresses equal to 9.5 , 9.6, 8.5 and 12.45 under $x$-, $y$-, equi- 
biaxial and shear loadings makes a reasonable choice for all types of in plane loading.

- Angle ply laminate [45/-45]s has produced 14, 14, and 28 under $\mathrm{x}-, \mathrm{y}$, and equi-biaxial loadings while it is 11.57 under shear loading which makes it unsuitable for general all types of loading

It is preferable to use [0/90]s laminates for square hole with normal sides under all types of in plane loading.

\section{References}

1. A. K. Kaw, Mechanics of Composite Materials, (Taylor \& Francis Group, LLC, 2006).

2. V. V. Vasiliev, E. V. Morozov, Mechanics and Analysis of Composite Materials, (Elsevier Science Ltd, 2001)

3. I. Dupir, N. Țăranu, A. Axinte "Effect of stacking sequence on the coefficients of mutual influence of composite laminates" (IManEE 2016).

4. A.Cutolo,A.R.Carotenuto,S.Palumbo,L.Esposito, V.Minutolo,M.Fraldi,E.Ruocco, Meccanica 56, 1555 (2021)

5. Abdelhak K., Abdelouahab T., Mohamed O, Abdelhamid G., Finite Element Analysis of stress concentrations in Isotropic and composite plates with Elliptical holes, (Springer, Swizerland2015)

6. Wang,H.,Qin, Acta Mechanica, 223,1323 (2012).

7. Rameshwari, V. L., Kamble, D.N., Analysis of stress concentration of Laminated Composite plate with circular Hole (ISSN: 2394-3696 volume 1, Dec 2014)

8. Rao D.K.N., Babu M.R., Reddy K.R.N., Sunil D., Composite Structures, 92,2845 (2010)

9. R. T. Schwartz, H. S. Schwartz, AIAA Journal, 5, 2, (1967)

10. Zeaid H., Tooling for Composite Aerospace Structures, (Butterworth-Heinemann,2020).

11. V. Krukonis "Boron and Refractory Borides" (1977).

12. Aditya Kumar,Akshay Agrawal, Ranjan Ghadai and Kanak Kalita. Science direct procedia Technology, 23, 156 (2016).

13. Wu, Hwai-Chung, and Bin Mu. Composites Part B: Engineering 34, 127 (2003).

14. Adams K. "The effect of cutouts on strength of GRP for naval ship hulls". MS Thesis. Department of Materials Science and Engineering, MIT (1986).
15. J.Rezaeepazhand,M.Jafari. Composite Structures 71, 463 (2005).

16. A.J. BALL, R. YOUNG, A. CERVENKA. Developments in the Science and Technology of Composite Materials (1990).

17. N. J. Pagano, R. Byron Pipes. J. Composite Materials, 5 (2014).

18. Peters S T Handbook of composites Second edition Cambridge University Press (Great Britain: Chapman \& Hall) 1998.

19. S. C. Amico, C. C. Angrizani, M. L. Drummond, J. Reinf. Plast. Compos., 29179 (2010).

20. Khechai A, Tati A, Belarbi MO, Guettala A. InDesign and Modeling of Mechanical Systems427 (2015).

21. Wang,H.,Qin,Acta Mechanica 223(6),1323 (2012).

22. Ramesh W, Vivek L. International Journal Of Innovations In Engineering Research And Technology. 1(2), (2014)

23. Mohammadi M, Dryden JR, Jiang L. International Journal of Solids and Structures 48(3-4), 483 (2011)

24. . Gao, X.-L., Int. Journal of Pressure Vessels and Piping, 67, 95 (1996).

25. Hufenbach W. Schaffer M. and Herrmann A.S. "Calculation of the Stress and Displacement Field of Anisotropic Plates with Elliptical Hole",507517,60,(1990).

26. Hufenbach W, Schaefer M, Herrmann AS. Archiv.60(8), 507 (1990).

27. Dinesh Kumar, S.B. Singh, Composites Part B: Engineering, 43, 142 (2012).

28. Jong T.D. , Journal of Composite Materials, 15, 311(1981). 